\title{
Optimal Motion of a Motor Cycle Rider on Sudden Halt
}

\author{
Chandan Chattoraj ${ }^{1}$ \\ Dr.B.C.Roy Engineering College, Durgapur, West Bengal, India
}

\begin{abstract}
In this paper, a new concept has been proposed to theoretically analyze the forces and torques on the upper body of a human riding on a two wheeler while facing a sudden halt/crash. This approach is applied to different joints by using a musculoskeletal model of the upper body. The optimal motion of the upper body during the sudden halt/crash is obtained by converting the multi degree of freedom control problem to a parameter based problem to study the nature and modes of response following a shock. A mathematical model with reduced degrees of freedom is proposed that retains the essential features of the motion most probable in the direction of the original velocity and the same (a non-linear formulation) is numerically investigated to find out the forces and torques on the thorasic trunk and the head-neck with the waist joint together with the displacements.
\end{abstract}

Keywords: Biomechanics, Muscles and skeleton, Link, Joints, Nonlinear stiffness, Control.

\section{Introduction}

It is very significant to analyze the loads in the various parts of the body of a human rider when his two wheeler experiences a sudden halt. This situation typically arises in a crash. Considering the increasing number of two wheelers on the roads and their availability to the younger generation $[8,9,13]$ worldwide today, it becomes necessary to theoretically analyze the damaging effect that such crashes can inflict on the rider. Especially, the acceleration of the brain is of greatest importance. While sustained fluctuating accelerations greater than $\pm 1 \mathrm{~g}$ is deleterious to the subject so far as his control over the nervous system is concerned, shock acceleration greater than $15 \mathrm{~g}$ is tantamount to brain concussion leading even to loss of life. It is also questionable as to whether the use of a helmet (that increases the inertia of the head) is beneficial when the impulse is received through the neck instead of a direct concussion of the head against a harder object.

A similar problem has been addressed by Wang et al [1] while they consider the loads on the lower limb joints of a human being jumping vertically down from a given height. Their intent was to device means to minimize the forces on the toe, the ankle and the knee joints by what they call an optimal motion. Prior to Wang several workers like Moeinzadeh [4] and Mizrahi [3] have attempted such studies from the angles of dynamic modelling. It is pertinent to mention that analysis of parameters that affect impact response have also been given a prime consideration [11,12,14]. Wang et al however dealt with the dynamic analysis of a human model - more eruditely, the musculoskeletal model by taking into consideration active motion of the muscles. The nuance in their work lies in converting the multi degrees of freedom and non-linear optimal control problem to one of parameter optimization.

It is well known to that the musculoskeletal structure of the spine that consists of a number of vertebrae reduces to one of many degrees of freedom that execute three-

* Corresponding author: Chandan Chattoraj, E-mail address: chandan.chattoraj@bcrec.ac.in 
dimensional translation as well as rotation $[5,6]$. The problem is further complicated by the behavior of the muscles $[7,10]$. Wang et al have considered the stiffness of the muscles as non-linear and fitted a cubic hardening characteristic with the displacement. The muscles are non-linear visco-elastic elements and damping is built into their structure.

The idea of reduction of degrees of freedom as adopted by Wang et al has influenced the present authors to propose their model. In this case the rider is assumed to be moving with his machine horizontally at a certain given velocity and a sudden stoppage of the same results. It can be justifiable to consider the upper thorasic trunk containing the major part of the spinal cord as a rigid bar executing angular motion about the hip joints. This immediately rules out many degrees of freedom; secondly the head can be considered as a massive body executing angular motion relative to shoulder neck joint. The part of the spines in this case can be considered as a mass less rigid rod. The problem is thus reduced to one or two degrees of freedom with generalized coordinates $\theta_{1}$ and $\theta_{2}$ as shown in figure 1. Furthermore, the two arms holding on to the handle bar have been made to represent a mass less spring damper connection. We can now define our intent in simple terms as follows:

Given a crash velocity what must be the optimum parameters $\mathrm{C}_{1}, \mathrm{~K}_{1}, \mathrm{C}_{2}, \mathrm{~K}_{2}, \mathrm{C}_{3}, \mathrm{~K}_{3}$, such that the following are optimized:-
a) The torque at the shoulder neck joint
b) The torque at the waist femur joint
c) The force in the arms
d) Acceleration of the brain matter
e) Rotations (must be finite and small)

\section{Musculoskeletal model of the upper body}

Since the thorasic trunk and the head-neck with the waist joint of a human rider on his two wheeler experiences (absorbs) shock during a sudden halt or a crash, it is indispensable to establish a musculoskeletal model of the upper body for an estimation of forces on it. In the present study, the authors consider the thorasic trunk and the head-neck with the waist joint and the neck provided with lumped inertia, damping and stiffness. The arms are considered as spring damper supports which bear against the handle bar [Fig1]. The study considers cubic hardening characteristics of the stiffness (muscle fibers) and the lower limb (below the waist) is assumed to be fixed with the machine of the rider.

\section{Mathematical Model}

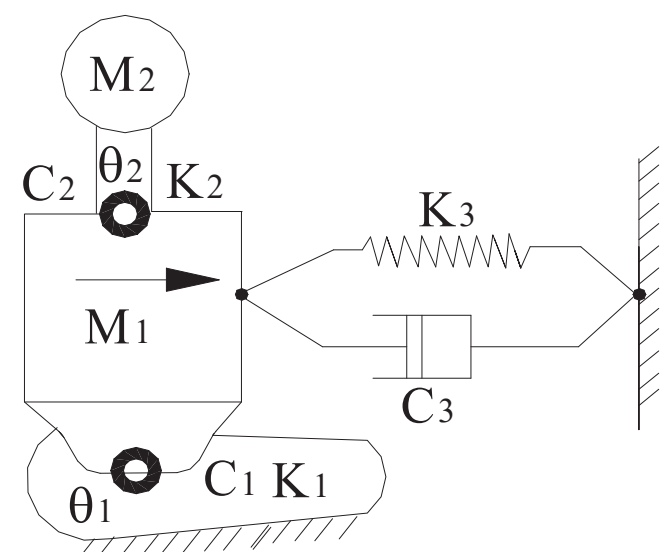

Figure 1: Musculoskeletal model of the upper body

In figure 1, the descriptions of the used notations are as following:

a: length of the head-neck

$b$ : length of the thorasic trunk

$h$ : height of the arm above the hip joint

$M_{1}$ : mass of the thorasic trunk

$M_{2}$ : equivalent translating mass of the head.

$C_{1}$ : viscous damping coefficient at the hip

$C_{2}$ : viscous damping coefficient at the head-neck

$c_{3}$ : viscous damping coefficient at the arm.

$K_{i}$ : spring constant at the waist

$K_{2}$ : spring constant at the head-neck

$K_{3}$ : spring constant at the arm.

$\theta_{i}$ : absolute angular displacement at the hip joint

$\theta_{2}$ : relative angular displacement at the head-neck.

$\phi=$ dissipation function

$T=$ kinetic energy function

$U=$ potential energy function

From figure 1,

$$
\begin{aligned}
& T=\frac{1}{2} \times \frac{1}{3} M_{1} \cdot b^{2} \dot{\theta}_{1}+\frac{1}{2} M_{2}\left[a \cdot \dot{\theta}_{1}+b \cdot \dot{\theta}_{2}\right]^{2} \\
& \phi=\frac{1}{2} \cdot C_{1} \cdot \dot{\theta}_{1}^{2}+\frac{1}{2} \cdot C_{3} \cdot\left(h \cdot \dot{\theta}_{1}\right)^{2}+\frac{1}{2} \cdot C_{2}\left(\dot{\theta}_{1}-\dot{\theta}_{2}\right)^{2} \\
& U=1 / 2 \cdot\left(K_{1} \cdot \theta_{1}\right)+1 / 2 \cdot K_{2} \cdot\left(\theta_{1}-\theta_{2}\right)^{2}+1 / 2 \cdot K_{3}\left(h \cdot \theta_{1}\right)^{2}
\end{aligned}
$$

The Lagrangian equations are:

$$
\frac{d}{d t} \cdot \frac{\partial T}{\partial \dot{\theta}_{r}}+\frac{\partial \varphi}{\partial \dot{\theta}_{r}}+\frac{\partial U}{\partial \theta_{r}}=0 ; \quad r=1,2 .
$$


Solving the above equation the final expressions obtained are

$\ddot{\theta}_{1}=\frac{3}{M_{1} b^{3}}\left[\begin{array}{l}-\left(C_{1} b+C_{3} h b+C_{2} b+C_{2} a\right)+\left(C_{2} b+C_{2} a\right) \dot{\theta}_{2} \\ -\theta_{1}\left(K_{1} b+K_{3} b h+K_{2} b+K_{2} a\right)+\theta_{2}\left(K_{2} b+K_{2} a\right)\end{array}\right]$

$\ddot{\theta}_{2}=\frac{1}{M_{2} b^{2}}\left[-M_{2} b a \ddot{\theta}_{1}-C_{2} \ddot{\theta}_{2}+C_{2} \theta_{1}-K_{2} \theta_{2}+K_{2} \theta_{1}\right]$

Where, $\mathrm{f}_{1}=$ absolute angular acceleration at the waist; $\mathrm{V}_{1}=$ absolute angular velocity at the waist; $\mathrm{f}_{2}$ $=$ relative angular acceleration at the head-neck; $V_{2}$ $=$ relative angular velocity at the head-neck

Following Wang et al, we assume a cubic hardening character for the muscles as follows (figure 1)

$$
\begin{aligned}
& K_{1}=\alpha_{1}+\beta_{1} \cdot \theta_{1}^{2} \\
& K_{2}=\alpha_{2}+\beta_{2}\left(\theta_{1}-\theta_{2}\right)^{2} \\
& h \cdot K_{3}=\alpha_{3}+\beta_{3} \cdot \theta_{1}^{2}
\end{aligned}
$$

\section{Forces and Torques on the muscles and Joints}

A human rider when on his/her two-wheeler experiences a sudden halt or a crash the deformed/ deflected musculoskeletal model of the upper body is shown in figure 2.

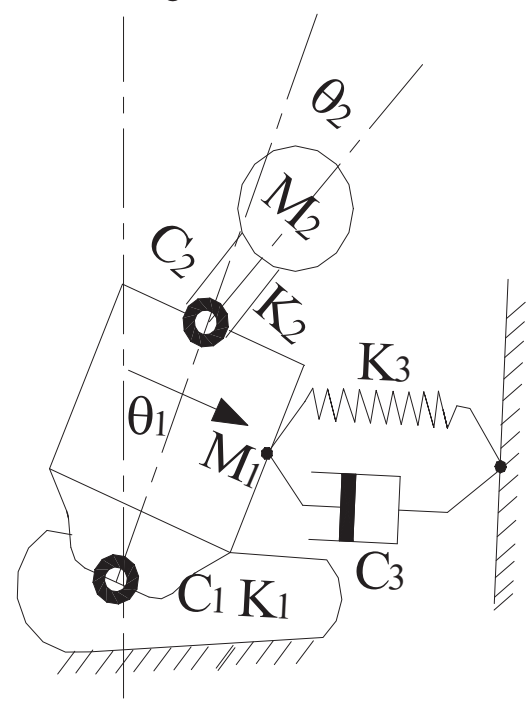

Figure 2: Exeggerated deflection pattern of the musculoskeletal model

The forces and torque on the thorasic trunk and the head neck joints can be visualized out from the deformed model and quantified by the following relations:

Torque at the head-neck joint

$\left(\tau_{2}\right)=K_{2} \cdot \theta_{2}+C_{2} \cdot V_{2}$

Torque at the waist joint

$\left(\tau_{1}\right)=K_{1} \cdot \theta_{1}+C_{1} \cdot V_{1}$

Force at the waist joint

$\left(F_{3}\right)=K_{3} h \theta_{1}+C_{3} h V_{1}$

Linear acceleration of the brain

$\left(f_{b}\right)=b \ddot{\theta}_{1}+a \ddot{\theta}_{2}$

\section{Results of Simulation (following Runge Kutta algorithms)}

Please refer to the figure 3 to 8 for various results
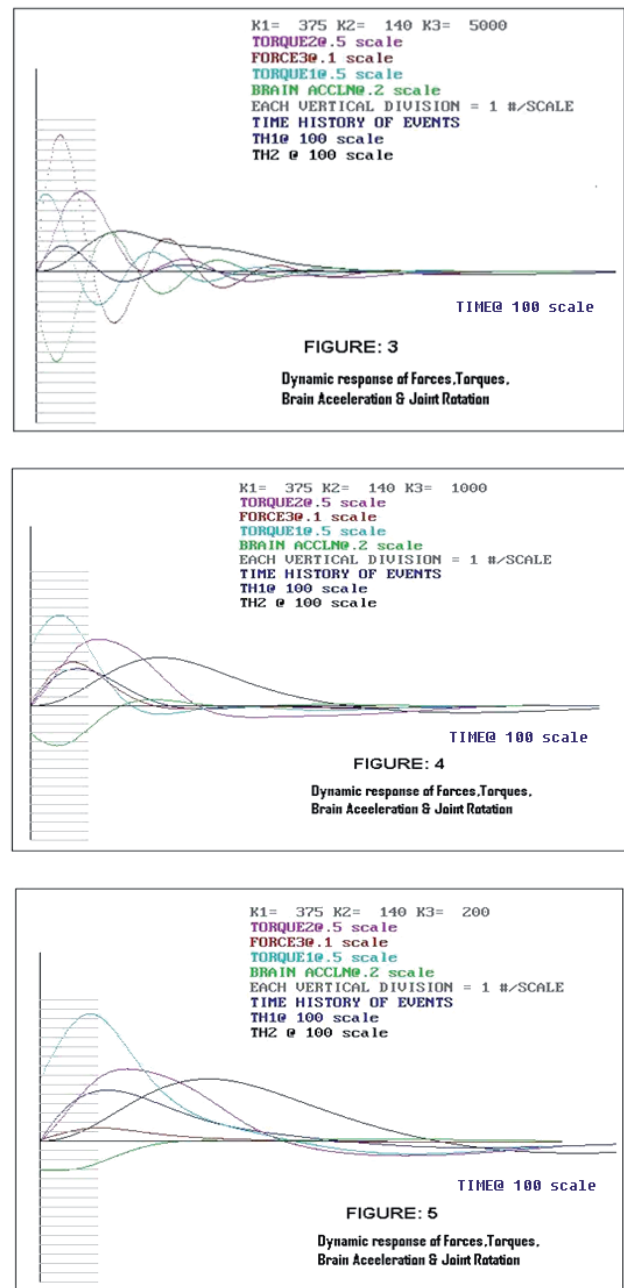

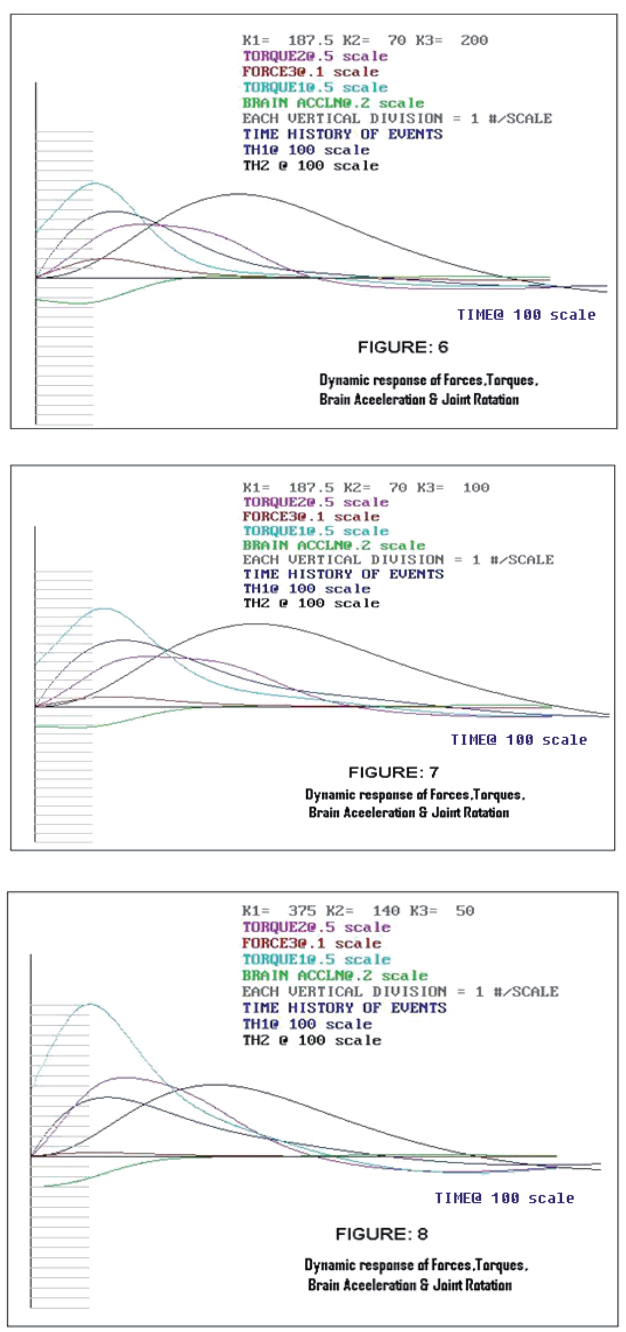

\section{Results discussion and conclusion}

The following are the basic data for the human model chosen for our analysis.

For all cases $\alpha_{1}=\beta_{1}=\mathrm{K}_{1} ; \alpha_{2}=\beta_{2}=\mathrm{K}_{2} ; \alpha_{3}=$ $\beta_{2}=\mathrm{K}_{3}$ (following Wang et al)

$a=0.2 m ; b=0.5 m ; h=0.4 m ; M_{1}=17 \mathrm{~kg} ; M_{2}=$ $3.1 \mathrm{~kg}$

$$
\begin{aligned}
C_{1} & =0.4 \times \sqrt{\frac{3 K_{1}}{M_{1} \cdot b^{2}}} \\
C_{2} & =0.4 \times \sqrt{\frac{K_{2}}{M_{2} \cdot a^{2}}} \\
C_{3} & =0.4 \times \sqrt{\frac{K_{3}}{M_{1}}}
\end{aligned}
$$

These correspond to a damping ratio of $20 \%$ considering the systems as individual and uncoupled.

Table 1

\begin{tabular}{|l|l|l|l|l|l|l|}
\hline $\begin{array}{l}\text { Figure } \\
\text { No. }\end{array}$ & $\begin{array}{l}\mathrm{K}_{1} \\
(\mathrm{Nm} / \mathrm{rad})\end{array}$ & $\begin{array}{l}\mathrm{K}_{2} \\
(\mathrm{~N} / \mathrm{m})\end{array}$ & $\begin{array}{l}\mathrm{K}_{3} \\
(\mathrm{Nm} / \mathrm{rad})\end{array}$ & $\begin{array}{l}\text { Brain } \\
\text { accelerati- } \\
\text { on. (fb) }\end{array}$ & $\begin{array}{l}\theta_{1} \\
\text { (in de- } \\
\text { grees) }\end{array}$ & $\begin{array}{l}\theta_{1} \\
\text { (in de- } \\
\text { grees) }\end{array}$ \\
\hline 3 & 375 & 140 & 5000 & 459 & 1.5 & 2.5 \\
\hline 4 & 375 & 140 & 1000 & 239 & 2.5 & 3.4 \\
\hline 5 & 375 & 140 & 200 & 169 & 3.2 & 4.2 \\
\hline 6 & 187.5 & 70 & 200 & 139 & 4.2 & 5.4 \\
\hline 7 & 187.5 & 70 & 100 & $11 \mathrm{~g}$ & 4.5 & 4.8 \\
\hline 8 & 375 & 140 & 50 & 159 & 3.6 & 4.6 \\
\hline
\end{tabular}

The results of computer simulation with varying stiffness parameters are well documented in fig. 3 to fig. 8 which shows the Time History of the important events like forces, torques, rotations and brain acceleration. The stiffness parameters of the biological springs (i.e. the muscles) are variable within wide ranges as the human specimen relaxes or stiffens his muscles. The hardening parameter, following Wang et al. , have been assumed to be equal to the basic value of the stiffness at zero displacement.

The results corroborate the facts that stiffening the muscles unduly during an anticipated crash can be more damaging. It is particularly important to realize that the stiffness of the hip (thigh) joint should be kept low to allow a higher compliance. The following values of the $\mathrm{K}$ parameters are found to be safe and satisfactory for the human model chosen for the analysis, where the crash velocity is standardized at $36 \mathrm{~km} / \mathrm{hr}(10 \mathrm{~m} / \mathrm{s})$ :

$$
\mathrm{K} 1=187.5 \mathrm{Nm} / \mathrm{rad} ; \mathrm{K} 2=70=\mathrm{N} / \mathrm{m} ; \mathrm{K} 3=100 \mathrm{Nm} / \mathrm{rad} \text {. }
$$

\section{Conclusions}

The present analysis is rather simplistic though the same maintains the essential features of the object of study. It is also important to investigate the configurations attained by the human model when the crash induces a twist and a vertical motion, which were altogether disregarded in this analysis. Further, a more detailed analysis considering masses of the vertebrae and the stiffness of the associated muscles will improve the study. Also, further investigation of the stiffening effects of the muscles, their damping and hardening characteristics and their inter relation with blood pressure remains open for study. 


\section{Acknowledgement}

The authors are highly indebted to the authorities of Dr. B. C. Roy Engineering College, Durgapur for their kind support.

\section{References}

1. WANG Yongjin., MATSUHISA Hiroshi., HONDA Yoshihisa, Loads on Lower Limb Joints and Optimal Action of Muscles for Shock Reduction, JSME International Journal., Series C, Vol. 42, No.3, 1999

2. Goh,C.J and Teo, K.L., Control Parameterization: A Unified Approach to Optimal Control Problems with General Constraints, Automatica, Vol. 24, No.1 (1988), p. 3 - 18.

3. Mizrahi, J. and Susak, Z., Analysis of Parameters Affecting Impact Force Attenuation during Landing in Human Vertical Free Fall, Engineering in Medicine, Vol. 11, No. 3(1982), p.141 $-147$.

4. Moeinzadeh. M,H., Engin, A.E. and Akkas, N., Two Dimensional Dynamic Modelling of Human Knee Joints, J. Biomechanics, Vol. 16, No. 4(1983), p.253 - 264.

5. Khandpur, R.S., Handbook of Biomedical InstrumentationSecond Edition, Tata McGraw Hill Publishing Company Limited, New Delhi.

6. Guyton and Hall, Text Book of Medical Physiology., Harcourt India Private Limited.

7. Mustafa Talib Yousif, Ahmad Farhan Mohd Sadullah, Khairil Anwar Abu Kassim: A review of behavioural issues contribution to motorcycle safety, IATSS Research, Volume 44, Issue 2, 2020, Pages 142-154, ISSN 0386-1112, https:// doi.org/10.1016/j.iatssr.2019.12.001.

8. S. Harnen, R. S. Radin umar, et al: Motorcycle Crash Prediction Model For Non-Signalized Intersections, IATSS RESEARCH Vol.27 No.2, 2003

9. Hassan, Omayma \& Shaker, Raneem \& Eldesouky, Rasha \& Hasan, O. \& Bayomy, Hanaa. (2014). Motorcycle Crashes: Attitudes of the Motorcyclists Regarding Riders'Experience and Safety Measures. Journal of Community Health. 39. 10.1007/s10900-014-9883-1.

10. Sapkota D, Bista B and Adhikari SR (2016) Economic Costs Associated with Motorbike Accidents in Kathmandu, Nepal. Front. Public Health 4:273. doi: 10.3389/fpubh.2016.00273.

11. Filbert Francis, Candida Moshiro, Berg Hans Yngve \& Marie Hasselberg (2021) Investigation of road infrastructure and traffic density attributes at high-risk locations for motorcycle-related injuries using multiple correspondence and cluster analysis in urban Tanzania, International Journal of Injury Control and Safety Promotion, DOl: 10.1080/17457300.2021.1930060.

12. Julaluk Carmai, Saiprasit Koetniyom \& Wasif Hossain (2019) Analysis of rider and child pillion passenger kinematics along with injury mechanisms during motorcycle crash, Traffic Injury Prevention, 20:sup1, S13-S20, DOI: 10.1080/15389588.2019.1616180.

13. Mullin B, Jackson R, Langley J, et al: Increasing age and experience: are both protective against motorcycle injury? A case-control study Injury Prevention 2000;6:32-35.

14. Cavalcanti A. L, Ferreira F. H. C, Olinda R. A, Padilha W. W. N, Cavalcanti A. F. C. Motorcycle-Related Cranio-Maxillofacial Injuries Among Brazilian Children and Adolescents. Biomed Pharmacol J 2017;10(4). 